\title{
Genetic Association between Foliage Yield and Contributing Traits in Vegetable Chenopods: Implications for Genetic Improvement
}

\author{
Atul BHARGAVA ${ }^{1,2 *}$, Francisco FUENTES ${ }^{3}$, Sudhir SHUKLA ${ }^{2}$, \\ Shilpi SRIVASTAVA ${ }^{1}$, Deepak OHRI ${ }^{2,4}$ \\ ${ }^{1}$ Amity Institute of Biotechnology, Amity University Uttar Pradesh (Lucknow Campus), Lucknow 226028, \\ India; abhargava@amity.edu (*correspondingauthor); ssrivastaval@lko.amity.edu \\ ${ }^{2}$ National Botanical Research Institute, Division of Genetics and Plant Breeding, Lucknow 226001, \\ India;s_shukla31@rediffmail.com;dohri@lko.amity.edu \\ ${ }^{3}$ Pontificia Universidad Católica de Chile, Facultad de Agronomía e Ingeniería Forestal, \\ Vicuña Mackenna 4860, Macul, Santiago, Chile; frfuentesc@uc.cl \\ ${ }^{4}$ Amity University Uttar Pradesh (Lucknow Campus), Research Cell, Lucknow 226028, India
}

\begin{abstract}
A two-year study was conducted to evaluate the foliage yield potential in 13 germplasm lines of Chenopodium album for 3 successive cuttings. Correlations among foliage yield and its contributing traits, along with path analysis was also worked out. Foliage yield was maximum for C. album IC 107297, followed by C. album H.P. and C. album amaranticolor. The genotype $\times$ year interaction was non-significant for all the traits except stem diameter and moisture content. Leaf size, plant height and stem diameter showed significant positive correlation with foliage yield both at phenotypic and genotypic levels in all the cuttings. Chlorophyll $a$ and chlorophyll $b$ showed positive association with carotenoid content and negative association with ascorbic acid in all the cuttings as well as on pooled basis. Significant negative association was observed between leaves/plant and foliage yield at genotypic level in all the cuttings ( $\mathrm{I}^{\text {st }}$ cutting: $-0.472^{*} ; \mathrm{II}^{\text {nd }}$ cutting: $-0.414^{*}$; $\mathrm{III}^{\text {rd }}$ cutting: $-0.480^{*}$ ) as well as on pooled basis $\left(-0.591^{* *}\right)$. Protein content negatively affected foliage yield in all the cuttings. Fibre content had high negative value of direct path for pooled data but positively influenced foliage yield indirectly via leaves/plant, stem diameter, chlorophyll $a$, chlorophyll $b$ and protein content. Ascorbic acid positively affected yield in $\mathrm{I}^{\text {st }}$ cutting as well as on pooled basis. Leaf size had high positive direct effect and significant positive association with foliage yield that indicates a true relationship between these traits. Leaf size also indirectly affected foliage yield in a positive direction through majority of other traits. Thus, direct selection for leaf size should be exercised to bring about improvement in foliage yield in C. album.
\end{abstract}

Keywords: Chenopodium album; foliage yield; protein; correlation; path analysis

\section{Introduction}

The increasing population of the world demands an increase in food production and cultivation of crops that are nutritious and require minimum inputs (Bhargava et al. 2010). Nowadays much attention has been centered on the exploitation and utilization of unusual and underutilized plant material for food (Bhargava et al., 2007a; Fuentes and Paredes-Gónzalez, 2015). Green vegetables have long been recognized as the cheapest and most abundant source of protein, vitamins and minerals (Aletor et al., 2002; Shukla et al., 2006). In recent years chenopods have evoked interest, as a potential food crop for diversification of agriculture to newer areas, environmental sustainability and for combating the nutritional deficiency in many parts of the world (Jacobsen, 2003; Bhargava et al., 2006a; Bhargava and Ohri, 2015, 2016; Bazile et al., 2016). This underutilized crop does not require high inputs and can be easily grown on agriculturally marginal lands (Partap et al., 1998; Bhargava et al., 2003a; Fuentes and Bhargava, 2011). Chenopods are being cultivated in the watersheds of the Chenab, Ravi, Beas, Satluj and Yamuna rivers in the western Himalayas, and in the hilly areas of North Bengal, watershed of Teesta river and several states of north-eastern India (Joshi, 1991; Partap et al., 1998). Although only three species viz. $C$. quinoa, C. pallidicaule and C. berlandieri subsp. nuttalliae are cultivated (Bhargava et al., 2006b), the leaves and tender 
stems of numerous other species are consumed as food and fodder (Tanaka, 1976; Kunkel, 1984, Partap and Kapoor, 1985; Partap, 1990; Moerman, 1998; Partap et al., 1998). The foliage of Chenopodium constitutes an inexpensive and rich source of protein $(26-64 \mathrm{~g} / \mathrm{kg})$, carotenoids $(78-190$ $\mathrm{mg} / \mathrm{kg}$ ) and vitamin C (0.5-2.4 g/kg) (Prakash et al., 1993; Fuentes and Paredes-Gónzalez, 2015). Besides this, the plant is recognized as an important medicinal plant in various ancient texts as well as by ethnic communities in many regions of the world (Bakshi et al., 1999; Kirtikar and Basu, 2001; Singh et al., 2003). Thus, vegetable chenopods are gaining importance due to their nutritional superiority and their ability to grow in agriculturally marginal lands with low levels of external inputs (Bhargava et al., 2006a). This makes it a potential crop for future diversification of agriculture in various parts of the world (Bilalis et al., 2018).

Yield is a complex quantitative measure being affected by genetic and environmental factors as a result of which direct selection based on yield could be misleading. Most of the traits of interest to the breeders are complex and are the result of interaction of a number of components. Due to this reason, the breeder is interested in understanding the relationship between yield and its components for making the best use of these relationships in selection (Bhargava $e t$ al., 2008). Correlation coefficient analysis quantifies the relationship between a given pair of traits and is of prime importance in yield improvement. Correlations between different traits have three main causes viz. pleiotropy, linkage and environmental effects (Falconer, 1989; Chen and Lübberstedt, 2010). A pleiotropic gene causes variation in two or more traits when the gene is segregating and is the major cause of correlation in populations, which have mated at random for successive generations (Solovieff et al., 2013). Linkage causes transient correlations but is broken by recombination in some populations (Falconer, 1989). Environmental correlations show similarity or dissimilarity in the response of traits to a specific environment and therefore correlations between traits obtained in one environment are not much reliable in predicting the response of the same population in another environment. (Falconer, 1989; Aastveit and Aastveit, 1993; Manenti et al., 2016). Knowledge about the magnitude and sign (positive or negative) of genotypic correlation is important for understanding the relationship between traits and fitness in natural populations, for prediction of correlated responses to selection and for formulation of selection indices in breeding programmes (Bhargava et al., 2007b; Punzalan et al., 2014; Madrid et al., 2018). However, correlation alone does not reliably predict the success of selection because high correlation between two traits might be due to the influence of a third trait or a group of traits (Bizeti et al., 2004). The information derived from correlation coefficients should therefore be augmented by partitioning of correlation coefficients into direct and indirect effects using the path coefficient analysis (Sincik and Goksoy, 2014).

Path analysis, an extension of multiple regression (Streiner, 2005), has been widely used in crop breeding to determine the nature of relationships between yield and its contributing components and to identify components with significant effects on yield for potential use as selection criteria. Wright (1921) first used this approach to organize and graphically portray the relationships between predictor variables and response variable through a path diagram based on experimental results. Path analysis, also known as standardized partial-regression coefficient, partitions the correlation coefficients into direct and indirect effects and thereafter allows the separation of direct influence of each trait on yield from the indirect effects caused by mutual association among the traits themselves (Garcia del Morel et al., 2003). In agriculture, path analysis has been extensively used by breeders to assist in the identification of traits that are useful as selection criteria to improve crop yield (dos Santos et al., 2014; Mihretu et al., 2014; Sincik and Goksoy, 2014; Ranjbar et al., 2015; Khan et al., 2016; Siddiqi et al., 2016). Although considerable literature is available on correlation and path analysis in other foliage crops (Kaul et al., 1996; Young et al., 2000; Carpici and Celik 2010; Abel et al., 2017), a limited amount of work has been conducted in Chenopodium spp. (Risi and Galwey, 1989; Bhargava et al., 2003b) and that too is limited to grain chenopods. There is no study with regard to foliage yield in vegetable chenopods. Thus, the present investigation was undertaken to gain in-depth knowledge of the interrelationship among various morphological and quality traits in successive cuttings, and to elucidate the extent and direction (positive and negative) of direct and indirect influence of component characters over yield.

\section{Materials and Methods}

\section{Experimental site}

The experiment was conducted at the experimental field of National Botanical Research Institute (N.B.R.I.), Lucknow, India. The experimental site is situated at an altitude of $120 \mathrm{~m}$ above sea level at $26.5^{\circ} \mathrm{N}$ latitude and $80.5^{\circ} \mathrm{E}$ longitude. In the Indo-Gangetic Plains of North India there are two main crop seasons, summer (KharifMarch to July) and winter (Rabi- October to February). Chenopodium grows mostly during the rabi season during which the minimum and maximum temperature ranges from $2.5-19^{\circ} \mathrm{C}$ and $14-29^{\circ} \mathrm{C}$ respectively.

\section{Experimental material}

A large collection of Chenopodium spp. is being maintained at N.B.R.I that contains locally available as well as introduced germplasm lines of $C$. album. Some of them have been collected during many expeditions to the Himalayan region while others have been introduced. The experimental material comprised 13 germplasm lines of $C$. album of which 9 were hexaploid, 1 tetraploid and 3 diploid (Table 1).

\section{The experiment}

The material was evaluated for 2 successive years in a randomized block design with 3 replications. The plot size for each replication in each year was $4 \mathrm{~m}^{2}$ with 6 rows per plot, spaced $30 \mathrm{~cm}$ apart. The field was disc ploughed and then harrowed and raked to obtain a good seed bed before sowing. No chemical fertilizer, fungicide or insecticide was applied either before or during the experiment. Weeding followed by hoeing was done at an interval of 20 days during the crop season. Irrigation was applied as and when needed. 
Table 1. Germplasm lines, their ploidy level, chromosome number and origin

\begin{tabular}{|c|c|c|c|}
\hline Germplasm lines & Ploidy level & Chromosome number & Origin \\
\hline C. album PRC 9802 & - & - & Himachal Pradesh, India \\
\hline C. album IC 107297 & - & - & Himachal Pradesh, India \\
\hline C. album 'Mexico' & $4 \mathrm{x}$ & 36 & Mexico \\
\hline C. album (local red) & $2 \mathrm{x}$ & 18 & Lucknow, India \\
\hline C. album 'Siliguri' & $2 \mathrm{x}$ & 18 & Siliguri, India \\
\hline C. album amaranticolor & $6 \mathrm{x}$ & 54 & Himachal Pradesh, India \\
\hline C. album 'H.P.' & $6 \mathrm{x}$ & 54 & Himachal Pradesh, India \\
\hline C. album 605700 & $6 \mathrm{x}$ & 54 & Michigan, USA ${ }^{a}$ \\
\hline C. album CHEN 60/76 & $6 \mathrm{x}$ & 54 & Belgium $^{\mathrm{b}}$ \\
\hline C. album CHEN 95/97 & $6 x$ & 54 & Unknown $^{\mathrm{b}}$ \\
\hline C. album 'Czech' & $6 \mathrm{x}$ & 54 & Czech Republic \\
\hline C. album 'Iowa' & $6 \mathrm{x}$ & 54 & Iowa, USA \\
\hline C. album 'Chandanbathua' & $2 \mathrm{x}$ & 18 & India \\
\hline
\end{tabular}

The germplasm was sown as a winter crop around midNovember in both the years. In each year, 3 foliage cuttings were performed at an interval of 15 days starting after $3^{\text {rd }}$ week of sowing. Data was recorded on 5 randomly selected plants from each replication in each cutting for 5 morphological traits namely plant height $(\mathrm{cm})$, branches / plant, leaves/plant, leaf size $\left(\mathrm{cm}^{2}\right)$ and stem diameter $(\mathrm{cm})$, separately for each cutting. Foliage yield was recorded as fresh weight on plot basis for each of the 3 cuttings and pooled for total foliage yield. Besides this, 7-quality traits viz. leaf moisture (\%), chlorophyll $a\left(\mathrm{mg} \mathrm{g}^{-1}\right)$, chlorophyll $b\left(\mathrm{mg} \mathrm{g}^{-1}\right)$, carotenoid (mg 100 $\left.\mathrm{g}^{-1}\right)$, fibre (\%), protein (\%) and ascorbic acid (\%) were also estimated for individual cuttings from the bulked leaves of each replication. Chlorophyll $a$, chlorophyll $b$ and carotenoid was estimated in fresh leaves per the method proposed by Jensen (1978). Leaf protein was analysed in dried leaves following the method of Lowry et al. (1951), while ascorbic acid was analysed in fresh leaves as Glick (1954). Fibre content was estimated using the method proposed by Watson (1994).

\section{Statistical analysis}

The data were subjected to analysis of variance (ANOVA) as per Singh and Chaudhary (1985). The pooled mean values of both the experimental years were subjected to further statistical analysis. Genotypic and phenotypic correlations among different characters were analysed following Johnson et al. (1955). Path analysis (Dewey and Lu, 1959) was carried out to study the direct and indirect effects of dependent and independent variables on foliage yield. A trait was considered as effective if it showed significant positive correlation with yield, high positive direct effect and minimal negative indirect effect on yield.

\section{Results}

There were highly significant differences among the germplasm lines for most of the traits in individual cuttings as well as for pooled values except for chlorophyll $b$ in III $^{\text {rd }}$ cutting in Year 1 (range: $0.96-0.217 \mathrm{mg} \mathrm{g}^{-1}$ ), carotenoid in
II $^{\text {nd }}$ cutting in Year 2 (range: $8.32-17.16 \mathrm{mg} \mathrm{g}^{-1}$ ) and pooled data for leaf size in Year 2 (Table 2). These results indicate a high degree of variation for morphological as well as qualitative variation among the lines under study. The genotype $\times$ year interaction was non-significant for all the traits except stem diameter and moisture content (Table 2).

A perusal of foliage yield data (Table 3) revealed that mean foliage yield of 13 germplasm lines in both the years generally increased with successive cuttings and was maximum in the $\mathrm{III}^{\mathrm{rd}}$ cutting $\left(2.25+0.25 \mathrm{~kg}\right.$ plot $^{-1}$ and $\left.2.09+0.26 \mathrm{~kg} \mathrm{plot}^{-1}\right)$ (Table 3). Simultaneously, foliage yield increased in successive cuttings in each germplasm line in the year 1, except IC 107297, CHEN 60/76 and CHEN 95/97, which showed decrease after I $^{\text {nd }}$ cutting while, in year 2 only six germplasm lines showed increase in successive cuttings. Highest foliage yield for crop year 1 was recorded in IC $107297\left(3.13 \pm 0.42 \mathrm{~kg} \mathrm{plot}^{-1}\right)$, followed by 'H.P.' $\left(3.04 \pm 0.34 \mathrm{~kg} \mathrm{plot}^{-1}\right)$ and 'amaranticolor' $(2.56 \pm 0.30 \mathrm{~kg}$ plot $\left.^{-1}\right)$ while for year 2, IC $107297\left(3.03 \pm 0.39 \mathrm{~kg} \mathrm{plot}^{-1}\right)$ gave the highest yield, followed by 'H.P.' $(2.94 \pm 0.27 \mathrm{~kg}$ plot 1) and 'amaranticolor' $\left(2.41 \pm 0.20 \mathrm{~kg} \mathrm{plot}^{-1}\right)$.

Correlation analysis revealed that the values of genotypic correlation were generally higher than corresponding phenotypic values for most of the traits (Table 4). Leaf size, plant height and stem diameter showed consistent positive significant correlation with foliage yield both at phenotypic and genotypic levels in all the cuttings. All these traits were strongly associated with foliage yield on pooled basis, phenotypically (leaf size: $0.866^{* *}$; plant height: $0.698^{* *}$; stem diameter: $0.641^{* *}$ ) as well as genotypically (leaf size: $0.894^{* *}$; plant height: $0.714^{* *}$; stem diameter: $\left.0.682^{* *}\right)$. At genotypic level, significant negative correlation was observed between leaves / plant and foliage yield in all the cuttings ( $\mathrm{I}^{\text {st }}$ cutting: $-0.472^{*}$; II ${ }^{\text {nd }}$ cutting: $-0.414^{*}$; III ${ }^{\text {rd }}$ cutting: $\left.-0.480^{*}\right)$ as well as on pooled basis $\left(-0.591^{* *}\right)$. Branches/plant showed significant positive association with leaves/plant in the first 2 cuttings (genotypic values $0.604^{* *}$ and $0.617^{* *}$ respectively) and on pooled basis (genotypic value $0.417^{*}$ ). The corresponding phenotypic values were also significant, albeit a little lower in comparison to genotypic values. It was observed that among all the quality traits only chlorophyll $a$ 
and $b$ showed consistent correlation with foliage yield in all the cuttings. Chlorophyll $a$ was positively associated with foliage yield in all the cuttings, both phenotypically ( $\mathrm{I}^{\mathrm{st}}$ cutting: $0.680^{* *}$; II ${ }^{\text {nd }}$ cutting: $0.455^{*}$; III $^{\text {rd }}$ cutting: $\left.0.474^{*}\right)$ and genotypically ( $\mathrm{I}^{\mathrm{st}}$ cutting: $0.741^{* *}$; $\mathrm{II}^{\text {nd }}$ cutting: $0.480^{*}$; III ${ }^{\text {rd }}$ cutting: $\left.0.484^{*}\right)$. Positive association also existed between chlorophyll $b$ and foliage yield that decreased with each successive cutting, but was significant in the first 2 cuttings. An interesting observation was that leaf size exhibited highly significant positive association with chlorophyll a and chlorophyll $b$ in all the cuttings and on pooled basis. Leaf moisture content was negatively associated with chlorophyll $a$, chlorophyll $b$ and carotenoid content in the $\mathrm{I}^{\mathrm{st}}$ cutting and on pooled basis. Chlorophyll $a$ and $b$ were positively correlated between themselves and with stem diameter in all the cuttings and on pooled basis. Chlorophyll $a$ and chlorophyll $b$ showed positive association with carotenoid content and negative association with ascorbic acid in all the cuttings as well as on pooled basis. The most striking results were obtained in relation to foliage yield and 3 major nutritional traits viz. carotenoid, protein and ascorbic acid. It was noticed that the association between foliage yield and these traits was positively significant in the $\mathrm{It}^{\mathrm{st}}$ cutting, then the association decreased and became positive but non-significant in I $^{\text {nd }}$ cutting and finally became negative in the III $^{\text {rd }}$ cutting. Fibre content (range: 7.68-15.82\%) showed least association with all other quality traits as well as with foliage yield.

Leaf size showed positive direct effect towards foliage yield in all the cuttings ( $\mathrm{I}^{\mathrm{st}}$ cutting: $0.183 ; \mathrm{II}^{\text {nd }}$ cutting: 0.280 ; III ${ }^{\text {rd }}$ cutting: 0.710) and on pooled basis (1.640) (Table 5). In contrast, leaves/plant exhibited negative path with foliage yield in all the cuttings $(-1.791,-0.339$ and -0.245 respectively) (Table 5). Stem diameter also exhibited direct negative path with foliage yield in all the cuttings, except in $\mathrm{II}^{\mathrm{nd}}$ cutting, however it was positively contributing to foliage yield via leaves/plant, moisture content, fibre and ascorbic acid. Branches/plant directly influenced foliage yield in all the cuttings except in $\mathrm{III}^{\mathrm{rd}}$ cutting, and was the only trait, which indirectly influenced all the quality traits positively (Table 5). Plant height, in spite of having significant genotypic correlation with foliage yield, showed direct negative effect in all the cuttings except in III $^{\text {rd }}$ cutting, as well as on pooled basis. Chlorophyll $a$ and chlorophyll $b$ positively contributed towards yield for pooled data, while carotenoid showed positive direct effect towards foliage

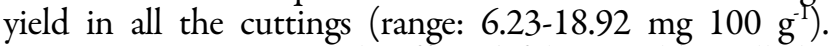
Protein content negatively affected foliage yield in all the cuttings (range: 2.62-5.29\%). Fibre content had high negative value of direct path for pooled data but positively influenced foliage yield indirectly via leaves/plant, stem

Table 2. Analysis of variance for different morphological and quality traits in vegetable Chenopodium (C. album L.) for 3 cuttings and pooled data over 2 years

\begin{tabular}{|c|c|c|c|c|c|c|c|c|c|}
\hline \multirow{2}{*}{ Traits/Years } & \multicolumn{2}{|c|}{$\mathrm{I}^{\mathrm{st}}$ Cutting } & \multicolumn{2}{|c|}{ II $^{\text {nd }}$ Cutting } & \multicolumn{2}{|c|}{ III $^{\text {rd }}$ Cutting } & \multicolumn{2}{|c|}{ Pooled data } & \multirow{2}{*}{$\mathrm{G} \times \mathrm{Y}^{\mathrm{a}}$} \\
\hline & Year 1 & Year 2 & Year 1 & Year 2 & Year 1 & Year 2 & Year 1 & Year 2 & \\
\hline Plant height $(\mathrm{cm})$ & $46.30^{* *}$ & $51.13^{* *}$ & $120.10^{* *}$ & $139.42^{* *}$ & $88.53^{* *}$ & $104.21^{* *}$ & $18.60^{* *}$ & $21.49^{* *}$ & 13.44 \\
\hline Branches/plant & $7.92^{* *}$ & $7.48^{* *}$ & $13.07^{* *}$ & $11.15^{* *}$ & $12.19^{* *}$ & $16.55^{* *}$ & $4.11^{* *}$ & $3.70^{* *}$ & 4.90 \\
\hline Leaves/plant & $114.50^{* *}$ & $133.17^{* *}$ & $155.24^{* *}$ & $178.19^{* *}$ & $81.46^{* *}$ & $121.24^{* *}$ & $39.15^{* *}$ & $44.16^{* *}$ & 21.13 \\
\hline Leaf size $\left(\mathrm{cm}^{2}\right)$ & $249.11^{* *}$ & $204.51^{* *}$ & $351.87^{* *}$ & $384.16^{* *}$ & $293.40^{* *}$ & $306.92^{* *}$ & $163.92^{* *}$ & 119.05 & 108.32 \\
\hline Stem diameter $(\mathrm{cm})$ & $0.034^{* *}$ & $0.012^{* *}$ & $0.021^{* *}$ & $0.016^{* *}$ & $0.029^{* *}$ & $0.016^{* *}$ & $0.012^{* *}$ & $0.017^{* *}$ & $0.027^{*}$ \\
\hline Moisture (\%) & $11.53^{* *}$ & $19.49^{* *}$ & $15.22^{* *}$ & $28.28^{* *}$ & $9.02^{* *}$ & $21.38^{* *}$ & $3.13^{* *}$ & $4.72^{* *}$ & $6.19^{*}$ \\
\hline Chlorophyll $a\left(\mathrm{mg} \mathrm{g}^{-1}\right)$ & $0.22^{* *}$ & $0.31^{* *}$ & $0.26^{* *}$ & $0.15^{* *}$ & $0.09^{*}$ & $0.12^{* *}$ & $0.06^{* *}$ & $0.10^{* *}$ & 0.06 \\
\hline Chlorophyll $b\left(\mathrm{mg} \mathrm{g}^{-1}\right)$ & $0.04^{* *}$ & $0.06^{* *}$ & $0.03^{* *}$ & $0.04^{* *}$ & 0.003 & $0.02^{* *}$ & $0.01^{* *}$ & $0.02^{* *}$ & 0.01 \\
\hline Carotenoid (mg 100g $\left.\mathrm{g}^{-1}\right)$ & $0.007^{* *}$ & $0.004^{* *}$ & $0.005^{* *}$ & 0.009 & $0.006^{* *}$ & $0.010^{* *}$ & $0.004^{* *}$ & $0.002^{* *}$ & 0.004 \\
\hline Fibre (\%) & $7.44^{* *}$ & $6.30^{* *}$ & $9.31^{* *}$ & $8.19^{* *}$ & $11.53^{* *}$ & $14.24^{* *}$ & $3.49^{* *}$ & $4.03^{* *}$ & 4.92 \\
\hline Protein (\%) & $0.94^{* *}$ & $0.79^{* *}$ & $0.32^{* *}$ & $0.42^{* *}$ & $0.46^{* *}$ & $0.41^{* *}$ & $0.32^{* *}$ & $0.39^{* *}$ & 0.24 \\
\hline Ascorbic acid (\%) & $0.005^{* *}$ & $0.004^{* *}$ & $0.004^{* *}$ & $0.004^{*}$ & $0.004^{* *}$ & $0.005^{* *}$ & 0.002 & $0.003^{* *}$ & 0.001 \\
\hline Foliage yield (kg/plot) & $1.39^{* *}$ & $1.53^{* *}$ & $2.41^{* *}$ & $2.30^{* *}$ & $2.84^{* *}$ & $2.76^{* *}$ & $2.03^{* *}$ & $2.24^{* *}$ & 1.17 \\
\hline
\end{tabular}

Table 3. Foliage yield of 13 germplasm lines of vegetable Chenopodium (C. album) for 2 successive years

\begin{tabular}{|c|c|c|c|c|c|c|c|c|}
\hline \multirow{2}{*}{ Genotype } & \multicolumn{4}{|c|}{ Year 1} & \multicolumn{4}{|c|}{ Year 2} \\
\hline & $\mathrm{I}^{\mathrm{st}}$ cutting & II $^{\text {nd }}$ cutting & IIII cutting & Mean & $\mathrm{I}^{\text {st }}$ cutting & II $^{\text {nd }}$ cutting & IIII ${ }^{\text {rd }}$ cutting & Mean \\
\hline C. album PRC 9802 & 0.8 & 2.4 & 3.35 & 2.18 & 0.69 & 2.17 & 3.64 & 2.16 \\
\hline C. album IC 107297 & 2.3 & 3.71 & 3.4 & 3.14 & 2.24 & 3.5 & 3.36 & 3.03 \\
\hline C. album 'Mexico' & 1.46 & 1.85 & 2.03 & 1.78 & 1.54 & 2.04 & 2.29 & 1.96 \\
\hline C. album (local red) & 0.98 & 1.2 & 1.64 & 1.27 & 1.11 & 1.13 & 0.88 & 1.04 \\
\hline C. album 'Siliguri' & 1.45 & 1.72 & 2.04 & 1.74 & 1.22 & 1.55 & 1.84 & 1.54 \\
\hline C. album amaranticolor & 1.96 & 2.8 & 2.94 & 2.57 & 2 & 2.66 & 2.58 & 2.41 \\
\hline C. album 'H.P.' & 2.35 & 3.31 & 3.48 & 3.05 & 2.48 & 3.43 & 2.91 & 2.94 \\
\hline C. album 605700 & 0.8 & 1.49 & 2.27 & 1.52 & 0.71 & 1.87 & 2.35 & 1.64 \\
\hline C. album CHEN 60/76 & 0.96 & 1.4 & 1.23 & 1.20 & 1.04 & 1.09 & 0.92 & 1.02 \\
\hline C. album CHEN 95/97 & 0.7 & 1.89 & 0.95 & 1.18 & 0.59 & 1.68 & 1.23 & 1.17 \\
\hline C. album 'Czech' & 0.39 & 0.64 & 0.78 & 0.60 & 0.24 & 0.4 & 0.58 & 0.41 \\
\hline C. album 'Iowa' & 2.16 & 2.6 & 2.72 & 2.49 & 2.06 & 2.37 & 2.09 & 2.17 \\
\hline C. album 'Chandanbathua' & 1.32 & 2.41 & 2.5 & 2.08 & 1.43 & 2.06 & 2.61 & 2.03 \\
\hline
\end{tabular}


Table 4. Phenotypic and genotypic correlation coefficients between foliage yield and its 5 agronomic and 7 quality traits in vegetable Chenopodium

\begin{tabular}{|c|c|c|c|c|c|c|c|c|}
\hline \multirow{2}{*}{ Characters } & \multicolumn{4}{|c|}{ Phenotypic } & \multicolumn{4}{|c|}{ Genotypic } \\
\hline & I & II & III & $\mathrm{P}$ & I & II & III & $\mathrm{P}$ \\
\hline \multicolumn{9}{|l|}{ Plant height vs } \\
\hline Branches/plant & 0.178 & $0.589^{* *}$ & $0.744^{* *}$ & $0.648^{* *}$ & 0.196 & $0.611^{* *}$ & $0.760^{* *}$ & $0.690^{* *}$ \\
\hline Leaves/plant & -0.230 & 0.277 & 0.071 & -0.021 & -0.249 & 0.303 & 0.093 & -0.018 \\
\hline Leaf size & 0.350 & $0.548^{* *}$ & $0.487^{* *}$ & $0.559^{* *}$ & 0.364 & $0.560^{* *}$ & $0.497^{* *}$ & $0.570^{* *}$ \\
\hline Stem diameter & -0.294 & $0.712^{* *}$ & 0.319 & $0.561^{* *}$ & -0.310 & $0.685^{* *}$ & 0.330 & $0.594^{* *}$ \\
\hline Moisture & 0.231 & -0.153 & $0.436^{*}$ & 0.224 & 0.245 & -0.170 & $0.453^{*}$ & 0.260 \\
\hline Chlorophyll a & -0.139 & $-0.449^{*}$ & 0.191 & 0.289 & -0.156 & $-0.460^{*}$ & 0.205 & 0.301 \\
\hline Chlorophyll b & -0.044 & $-0.547^{* *}$ & 0.269 & 0.220 & -0.059 & $-0.571^{* *}$ & 0.283 & 0.249 \\
\hline Carotenoid & -0.160 & -0.214 & -0.255 & 0.019 & -0.174 & -0.230 & -0.291 & 0.035 \\
\hline Fibre & $0.393^{*}$ & 0.219 & -0.054 & 0.024 & $0.410^{*}$ & 0.226 & -0.076 & 0.050 \\
\hline Protein & 0.020 & $0.439^{*}$ & $-0.431^{*}$ & $0.430^{*}$ & 0.049 & $0.460^{*}$ & $-0.449^{*}$ & $0.465^{*}$ \\
\hline Ascorbic acid & 0.246 & 0.081 & 0.024 & 0.199 & 0.265 & 0.105 & 0.070 & 0.240 \\
\hline Foliage yield & $0.429^{*}$ & $0.510^{* *}$ & $0.618^{* *}$ & $0.698^{* *}$ & $0.449^{*}$ & $0.581^{* *}$ & $0.653^{* *}$ & $0.714^{* *}$ \\
\hline \multicolumn{9}{|l|}{ Branches/plant vs } \\
\hline Leaves/plant & $0.578^{* *}$ & $0.589^{* *}$ & 0.183 & $0.389^{*}$ & $0.604^{* *}$ & $0.617^{* *}$ & 0.202 & $0.417^{*}$ \\
\hline Leaf size & 0.024 & 0.257 & 0.211 & 0.091 & 0.051 & 0.289 & 0.203 & 0.114 \\
\hline Stem diameter & 0.303 & 0.284 & -0.158 & 0.214 & 0.320 & 0.310 & -0.169 & 0.240 \\
\hline Moisture & $-0.569^{* *}$ & -0.120 & 0.379 & 0.008 & $-0.586^{* *}$ & -0.139 & $0.394^{*}$ & 0.026 \\
\hline Chlorophyll a & $0.521^{* *}$ & $-0.610^{* *}$ & -0.132 & 0.159 & $0.539^{* *}$ & $-0.634^{* *}$ & -0.130 & 0.164 \\
\hline Chlorophyll b & $0.499^{* *}$ & $-0.446^{*}$ & -0.080 & 0.178 & $0.530^{* *}$ & $-0.480^{* *}$ & -0.061 & 0.213 \\
\hline Carotenoid & $0.470^{*}$ & -0.090 & 0.101 & 0.231 & $0.429^{*}$ & -0.068 & 0.136 & 0.240 \\
\hline Fibre & -0.284 & -0.042 & 0.081 & 0.140 & -0.299 & -0.064 & 0.104 & 0.131 \\
\hline Protein & $0.502^{* *}$ & 0.248 & -0.126 & 0.360 & $0.519^{* *}$ & 0.232 & -0.147 & 0.378 \\
\hline Ascorbic acid & 0.069 & $0.510^{* *}$ & 0.194 & $0.461^{*}$ & 0.096 & $0.547^{* *}$ & 0.202 & $0.476^{*}$ \\
\hline Foliage yield & 0.297 & $0.421^{*}$ & 0.270 & 0.371 & 0.309 & $0.443^{*}$ & 0.293 & 0.348 \\
\hline \multicolumn{9}{|l|}{ Leaves/plant vs } \\
\hline Leaf size & $-0.420^{*}$ & $-0.384^{*}$ & $-0.490^{* *}$ & $-0.603^{* *}$ & $-0.439^{*}$ & -0.410 & $-0.511^{* *}$ & $-0.636^{* *}$ \\
\hline Stem diameter & 0.006 & -0.223 & $-0.594^{* *}$ & -0.340 & 0.017 & -0.240 & $-0.617^{* *}$ & -0.337 \\
\hline Moisture & $-0.440^{*}$ & -0.046 & $0.547^{* *}$ & -0.093 & $-0.451^{*}$ & -0.070 & $0.561^{* *}$ & -0.087 \\
\hline Chlorophyll a & -0.091 & -0.370 & $-0.436^{*}$ & -0.131 & -0.114 & -0.310 & $-0.460^{*}$ & -0.153 \\
\hline Chlorophyll b & -0.070 & -0.080 & -0.299 & 0.099 & -0.095 & -0.097 & -0.341 & 0.112 \\
\hline Carotenoid & 0.120 & $-0.399^{*}$ & -0.140 & -0.176 & 0.143 & $-0.424^{*}$ & -0.163 & -0.195 \\
\hline Fibre & $-0.464^{*}$ & -0.160 & 0.229 & -0.250 & $-0.480^{*}$ & -0.191 & 0.246 & -0.294 \\
\hline Protein & -0.191 & $0.439^{*}$ & $0.440^{*}$ & 0.180 & -0.214 & $0.460^{*}$ & $0.481^{*}$ & 0.197 \\
\hline Ascorbic acid & $-0.397^{*}$ & $0.449^{*}$ & $0.510^{* *}$ & 0.247 & $-0.410^{*}$ & $0.463^{*}$ & $0.591^{* *}$ & 0.260 \\
\hline Foliage yield & $-0.423^{*}$ & $-0.402^{*}$ & $-0.459^{*}$ & $-0.562^{* *}$ & $-0.472^{*}$ & $-0.414^{*}$ & $-0.480^{*}$ & $-0.591^{* *}$ \\
\hline \multicolumn{9}{|l|}{ Leaf size vs } \\
\hline Stem diameter & $0.523^{* *}$ & $0.729^{* *}$ & $0.684^{* *}$ & $0.860^{* *}$ & $0.536^{* *}$ & $0.760^{* *}$ & $0.710^{* *}$ & $0.874^{* *}$ \\
\hline Moisture & -0.027 & 0.159 & 0.173 & 0.123 & -0.040 & 0.178 & 0.201 & 0.149 \\
\hline Chlorophyll a & $0.590^{* *}$ & $0.621^{* *}$ & $0.635^{* *}$ & $0.569^{* *}$ & $0.620^{* *}$ & $0.702^{* *}$ & $0.754^{* *}$ & $0.675^{* *}$ \\
\hline Chlorophyll b & $0.619^{* *}$ & $0.637^{* *}$ & $0.670^{* *}$ & $0.617^{* *}$ & $0.646^{* *}$ & $0.650^{* *}$ & $0.691^{* *}$ & $0.633^{* *}$ \\
\hline Carotenoid & $0.503^{* *}$ & 0.260 & -0.329 & 0.080 & $0.531^{* *}$ & 0.246 & -0.346 & 0.104 \\
\hline Fibre & 0.196 & 0.120 & -0.040 & -0.016 & 0.183 & 0.114 & -0.096 & -0.043 \\
\hline Protein & 0.350 & -0.251 & -0.331 & -0.109 & $0.369^{*}$ & -0.240 & -0.374 & -0.126 \\
\hline Ascorbic acid & -0.069 & -0.153 & -0.120 & 0.149 & -0.109 & -0.165 & -0.104 & -0.180 \\
\hline Foliage yield & $0.701^{* *}$ & $0.740^{* *}$ & $0.890^{* *}$ & $0.866^{* *}$ & $0.740^{* *}$ & $0.769^{* *}$ & $0.865^{* *}$ & $0.894^{* *}$ \\
\hline \multicolumn{9}{|l|}{ Stem diameter vs } \\
\hline Moisture & -0.121 & 0.150 & -0.290 & -0.144 & -0.140 & 0.164 & -0.309 & -0.165 \\
\hline Chlorophyll a & $0.643^{* *}$ & $0.605^{* *}$ & $0.590^{* *}$ & $0.630^{* *}$ & $0.631^{* *}$ & $0.623^{* *}$ & $0.610^{* *}$ & $0.621^{* *}$ \\
\hline Chlorophyll b & $0.630^{* *}$ & $0.581^{* *}$ & $0.410^{*}$ & $0.596^{* *}$ & $0.680^{* *}$ & $0.614^{* *}$ & $0.484^{*}$ & $0.649^{* *}$ \\
\hline Carotenoid & $0.491^{*}$ & -0.190 & -0.173 & 0.281 & $0.475^{*}$ & -0.213 & -0.199 & 0.308 \\
\hline Fibre & -0.130 & 0.241 & -0.211 & -0.152 & -0.156 & 0.265 & -0.254 & -0.169 \\
\hline Protein & $0.547^{* *}$ & -0.053 & $-0.504^{* *}$ & 0.051 & $0.580^{* *}$ & -0.042 & $-0.539^{* *}$ & 0.074 \\
\hline Ascorbic acid & -0.161 & -0.314 & -0.294 & -0.324 & -0.190 & -0.334 & -0.270 & -0.349 \\
\hline Foliage yield & 0.243 & $0.579^{* *}$ & $0.653^{* *}$ & $0.641^{* *}$ & 0.280 & $0.624^{* *}$ & $0.680^{* *}$ & $0.682^{* *}$ \\
\hline \multicolumn{9}{|l|}{ Moisture vs } \\
\hline Chlorophyll a & $-0.452^{*}$ & -0.189 & $-0.446^{*}$ & $-0.574^{* *}$ & $-0.470^{*}$ & -0.162 & $-0.464^{*}$ & $-0.583^{* *}$ \\
\hline Chlorophyll b & $-0.403^{*}$ & -0.061 & -0.249 & $-0.519^{* *}$ & $-0.429^{*}$ & -0.103 & -0.273 & $-0.540^{* *}$ \\
\hline Carotenoid & $-0.740^{* *}$ & -0.094 & $-0.617^{* *}$ & $-0.499^{* *}$ & $-0.781^{* *}$ & -0.124 & $-0.640^{* *}$ & $-0.526^{* *}$ \\
\hline Fibre & $0.471^{*}$ & 0.008 & 0.316 & $0.610^{* *}$ & $0.485^{*}$ & -0.063 & 0.360 & $0.645^{* *}$ \\
\hline Protein & -0.291 & $-0.480^{*}$ & $-0.484^{*}$ & -0.261 & -0.320 & $-0.463^{*}$ & $0.480^{*}$ & -0.299 \\
\hline Ascorbic acid & -0.171 & 0.123 & 0.264 & 0.134 & -0.195 & 0.140 & 0.290 & 0.166 \\
\hline Foliage yield & $-0.391^{*}$ & -0.074 & 0.239 & 0.056 & $-0.414^{*}$ & -0.102 & 0.271 & 0.095 \\
\hline
\end{tabular}




\begin{tabular}{|c|c|c|c|c|c|c|c|c|}
\hline \multicolumn{9}{|l|}{ Chlorophyll $a$ vs } \\
\hline Chlorophyll $b$ & $0.934^{* *}$ & $0.920^{* *}$ & $0.758^{* *}$ & $0.872^{* *}$ & $0.956^{* *}$ & $0.911^{* *}$ & $0.802^{* *}$ & $0.910^{* *}$ \\
\hline Carotenoid & $0.741^{* *}$ & 0.219 & 0.169 & $0.637^{* *}$ & $0.760^{* *}$ & 0.202 & 0.193 & $0.680^{* *}$ \\
\hline Fibre & -0.183 & 0.054 & $-0.396^{*}$ & -0.303 & -0.164 & 0.090 & $-0.419^{*}$ & -0.340 \\
\hline Protein & $0.856^{* *}$ & -0.194 & $-0.578^{* *}$ & 0.104 & $0.901^{* *}$ & -0.220 & $-0.594^{* *}$ & 0.110 \\
\hline Ascorbic acid & 0.083 & $-0.656^{* *}$ & -0.139 & $-0.549^{* *}$ & 0.070 & $-0.690^{* *}$ & -0.161 & $-0.560^{* *}$ \\
\hline Foliage yield & $0.680^{* *}$ & $0.455^{*}$ & $0.474^{*}$ & 0.250 & $0.741^{* *}$ & $0.480^{*}$ & $0.484^{*}$ & 0.292 \\
\hline \multicolumn{9}{|l|}{ Chlorophyll $b$ vs } \\
\hline Carotenoid & $0.879^{* *}$ & 0.218 & 0.159 & $0.629^{* *}$ & $0.900^{* *}$ & 0.256 & 0.140 & $0.645^{* *}$ \\
\hline Fibre & -0.103 & -0.141 & -0.093 & -0.313 & -0.109 & -0.160 & -0.123 & $-0.386^{*}$ \\
\hline Protein & $0.870^{* *}$ & -0.271 & $-0.480^{*}$ & -0.029 & $0.853^{* *}$ & -0.299 & $-0.455^{*}$ & -0.080 \\
\hline Ascorbic acid & 0.290 & $-0.450^{*}$ & $-0.391^{*}$ & $-0.410^{*}$ & 0.320 & $-0.481^{*}$ & $-0.405^{*}$ & $-0.437^{*}$ \\
\hline Foliage yield & $0.658^{* *}$ & $0.648^{* *}$ & 0.293 & 0.083 & $0.708^{* *}$ & $0.624^{* *}$ & 0.269 & 0.105 \\
\hline \multicolumn{9}{|l|}{ Carotenoid vs } \\
\hline Fibre & -0.267 & 0.098 & -0.051 & -0.101 & -0.290 & 0.106 & -0.049 & -0.156 \\
\hline Protein & $0.690^{* *}$ & -0.248 & $0.443^{*}$ & 0.014 & $0.714^{* *}$ & -0.298 & $0.470^{*}$ & -0.054 \\
\hline Ascorbic acid & 0.282 & -0.351 & -0.164 & -0.098 & 0.321 & -0.377 & -0.195 & -0.114 \\
\hline Foliage yield & $0.575^{* *}$ & $0.393^{*}$ & $-0.469^{*}$ & 0.149 & $0.546^{* *}$ & $0.410^{*}$ & $-0.481^{* *}$ & 0.190 \\
\hline \multicolumn{9}{|l|}{ Fibre vs } \\
\hline Protein & -0.130 & $0.569^{* *}$ & 0.130 & $-0.449^{*}$ & -0.154 & $0.544^{* *}$ & 0.185 & $-0.492^{* *}$ \\
\hline Ascorbic acid & 0.173 & -0.281 & -0.057 & -0.140 & 0.211 & -0.319 & -0.041 & -0.179 \\
\hline Foliage yield & 0.149 & 0.350 & -0.143 & -0.082 & 0.156 & 0.379 & -0.194 & -0.127 \\
\hline \multicolumn{9}{|l|}{ Protein vs } \\
\hline Ascorbic acid & $0.439^{*}$ & 0.059 & 0.369 & $0.460^{*}$ & $0.459^{*}$ & 0.110 & 0.377 & $0.484^{*}$ \\
\hline Foliage yield & $0.611^{* *}$ & 0.020 & $-0.529^{* *}$ & 0.256 & $0.650^{* *}$ & 0.084 & $-0.619^{* *}$ & 0.290 \\
\hline \multicolumn{9}{|l|}{ Ascorbic acid vs } \\
\hline Foliage yield & $0.396^{*}$ & 0.149 & -0.014 & 0.176 & $0.417^{*}$ & 0.163 & -0.085 & 0.188 \\
\hline
\end{tabular}

Table 5. Path coefficient analysis for 5 agronomic and 7 quality traits of foliage yield in vegetable Chenopodium

\begin{tabular}{|c|c|c|c|c|c|c|c|c|c|c|c|c|c|c|}
\hline \multicolumn{2}{|c|}{ Characters } & $\begin{array}{l}\text { Plant } \\
\text { height }\end{array}$ & $\begin{array}{l}\text { Branch. } \\
\text { /plant }\end{array}$ & $\begin{array}{l}\text { Leaves } \\
\text { / plant }\end{array}$ & $\begin{array}{l}\text { Leaf } \\
\text { size }\end{array}$ & $\begin{array}{l}\text { Stem } \\
\text { diam. }\end{array}$ & $\begin{array}{c}\text { Moisture } \\
\text { content }\end{array}$ & $\begin{array}{c}\text { Chl. } \\
a\end{array}$ & $\begin{array}{c}\text { Chl. } \\
b\end{array}$ & $\begin{array}{c}\text { Carote- } \\
\text { noid }\end{array}$ & Fibre & Protein & $\begin{array}{c}\text { Ascorbic } \\
\text { acid }\end{array}$ & $\begin{array}{l}\text { Genotypic } \\
\text { correlation }\end{array}$ \\
\hline \multirow{4}{*}{$\begin{array}{l}\text { Plant } \\
\text { height } \\
(\mathrm{cm})\end{array}$} & I & -0.436 & -0.068 & 0.113 & -0.153 & 0.153 & -0.140 & 0.077 & 0.029 & 0.087 & -0.168 & -0.013 & -0.130 & $0.449^{*}$ \\
\hline & II & -0.274 & -0.170 & -0.066 & -0.138 & -0.184 & 0.040 & 0.127 & 0.364 & 0.058 & -0.069 & -0.090 & -0.031 & $0.581^{* *}$ \\
\hline & III & 0.369 & 0.280 & 0.031 & 0.168 & 0.124 & 0.181 & 0.084 & 0.113 & -0.084 & -0.030 & -0.162 & 0.013 & $0.653^{* *}$ \\
\hline & $\mathrm{P}$ & -0.765 & -0.541 & 0.030 & -0.436 & -0.441 & -0.196 & -0.240 & -0.199 & -0.025 & -0.014 & -0.330 & -0.140 & $0.714^{* *}$ \\
\hline \multirow{4}{*}{$\begin{array}{l}\text { Branches/ } \\
\text { plant }\end{array}$} & I & 0.277 & 1.650 & 0.980 & 0.026 & 0.480 & -1.140 & 0.849 & 0.860 & 0.721 & -0.582 & 0.810 & 0.131 & 0.309 \\
\hline & / II & 0.369 & 0.483 & 0.288 & 0.114 & 0.152 & -0.067 & -0.330 & -0.240 & -0.060 & -0.031 & 0.133 & 0.261 & $0.443^{*}$ \\
\hline & III & -0.180 & -0.220 & -0.060 & -0.041 & 0.041 & -0.087 & 0.031 & 0.037 & -0.034 & -0.019 & 0.034 & -0.064 & 0.293 \\
\hline & $\mathrm{P}$ & 1.151 & 1.685 & 0.649 & 0.161 & 0.390 & 0.084 & 0.281 & 0.428 & 0.490 & 0.221 & 0.601 & 0.746 & 0.348 \\
\hline \multirow{4}{*}{$\begin{array}{l}\text { Leaves/ } \\
\text { plant }\end{array}$} & I & 0.441 & -1.084 & -1.791 & 0.743 & -0.019 & 1.171 & 0.140 & 0.132 & -0.170 & 0.891 & 0.381 & 0.710 & $-0.472^{*}$ \\
\hline & II & -0.102 & -0.205 & -0.339 & 0.136 & 0.080 & 0.010 & 0.289 & 0.230 & 0.126 & 0.057 & -0.140 & -0.130 & $-0.414^{*}$ \\
\hline & III & -0.021 & -0.049 & -0.245 & 0.112 & 0.130 & -0.142 & 0.128 & 0.084 & 0.040 & -0.047 & -0.165 & -0.120 & $-0.480^{*}$ \\
\hline & $\mathrm{P}$ & 0.034 & -0.314 & -0.840 & 0.541 & 0.301 & 0.103 & 0.143 & -0.084 & 0.202 & 0.240 & -0.175 & -0.206 & $-0.591^{* *}$ \\
\hline \multirow{4}{*}{$\begin{array}{l}\text { Leaf } \\
\text { size } \\
\left(\mathrm{cm}^{2}\right)\end{array}$} & I & 0.106 & 0.032 & -0.070 & 0.183 & 0.096 & -0.009 & 0.109 & 0.104 & 0.102 & 0.050 & 0.070 & -0.014 & $0.740^{* *}$ \\
\hline & II & 0.146 & 0.076 & -0.117 & 0.280 & 0.211 & 0.039 & -0.049 & -0.099 & 0.071 & 0.048 & -0.081 & -0.035 & $0.769^{* *}$ \\
\hline & III & 0.365 & 0.152 & -0.350 & 0.710 & 0.483 & 0.129 & 0.254 & 0.205 & -0.244 & -0.033 & -0.281 & -0.094 & $0.865^{* *}$ \\
\hline & $\mathrm{P}$ & 0.940 & 0.163 & -1.042 & 1.640 & 1.430 & 0.183 & 0.540 & 0.371 & 0.130 & -0.026 & -0.160 & -0.210 & $0.894^{* *}$ \\
\hline \multirow{4}{*}{$\begin{array}{l}\text { Stem } \\
\text { diameter } \\
(\mathrm{cm})\end{array}$} & I & 0.172 & -0.163 & -0.021 & -0.253 & -0.463 & 0.034 & -0.322 & -0.329 & -0.236 & 0.081 & -0.264 & 0.097 & 0.280 \\
\hline & II & 0.149 & 0.073 & -0.051 & 0.153 & 0.186 & 0.036 & -0.030 & -0.090 & -0.059 & 0.053 & -0.016 & -0.064 & $0.624^{* *}$ \\
\hline & III & -0.040 & 0.020 & 0.098 & -0.112 & -0.163 & 0.064 & -0.113 & -0.070 & 0.031 & 0.041 & 0.079 & 0.053 & $0.680^{* *}$ \\
\hline & P & -0.825 & -0.316 & 0.537 & -1.245 & -1.452 & 0.244 & -0.970 & -0.870 & -0.488 & 0.296 & -0.039 & 0.480 & $0.682^{* *}$ \\
\hline \multirow{4}{*}{$\begin{array}{l}\text { Moisture } \\
\text { content } \\
(\%)\end{array}$} & I & 0.052 & -0.016 & -0.040 & -0.004 & -0.014 & 0.030 & -0.028 & -0.024 & -0.081 & 0.033 & -0.021 & -0.012 & $-0.414^{* *}$ \\
\hline & II & 0.063 & 0.027 & 0.011 & -0.057 & -0.053 & -0.271 & 0.252 & 0.271 & 0.031 & 0.021 & 0.143 & -0.028 & -0.102 \\
\hline & III & 0.010 & 0.013 & 0.022 & 0.009 & -0.014 & 0.048 & -0.021 & -0.024 & -0.024 & 0.014 & 0.004 & 0.020 & 0.271 \\
\hline & $\mathrm{P}$ & 0.182 & 0.032 & -0.070 & 0.080 & -0.120 & 0.702 & -0.502 & -0.481 & -0.513 & 0.465 & -0.194 & 0.091 & 0.095 \\
\hline \multirow{4}{*}{$\begin{array}{l}\text { Chl. a } \\
\left(\mathrm{mg} \mathrm{g}^{-1}\right)\end{array}$} & I & 0.074 & -0.301 & 0.049 & -0.306 & -0.394 & 0.328 & -0.536 & -0.514 & -0.430 & 0.097 & -0.440 & -0.034 & $0.741^{* *}$ \\
\hline & II & -0.090 & -0.140 & -0.079 & -0.050 & -0.048 & -0.059 & 0.203 & 0.321 & 0.056 & 0.020 & -0.040 & -0.140 & $0.480^{*}$ \\
\hline & III & -0.014 & 0.012 & 0.043 & -0.029 & -0.048 & 0.044 & -0.070 & -0.081 & 0.025 & 0.033 & 0.054 & 0.016 & $0.484^{* *}$ \\
\hline & $\mathrm{P}$ & 0.023 & 0.014 & -0.075 & 0.024 & 0.036 & -0.035 & 0.045 & 0.045 & 0.083 & -0.024 & 0.015 & -0.030 & 0.292 \\
\hline
\end{tabular}




\begin{tabular}{|c|c|c|c|c|c|c|c|c|c|c|c|c|c|c|}
\hline \multirow{4}{*}{$\begin{array}{l}\text { Chl. } b \\
\left(\mathrm{mg} \mathrm{g}^{-1}\right)\end{array}$} & I & -0.036 & 0537 & 0093 & 0633 & 063 & $05 \%$ & 096 & 0020 & & & & & \\
\hline & II & 0.325 & 0271 & 0.056 & 0203 & 0233 & 0029 & $0=2$ & 058 & & $0<0$ & $0,10 ?$ & & \\
\hline & UI & 0.016 & -0.008 & -0016 & 0.007 & 0.026 & -0.009 & 0.034 & 0.024 & 00 & וברז5 & 007 & 017 & 020 \\
\hline & $\mathrm{P}$ & 0.039 & 0.029 & 0.017 & 0.030 & 0.086 & -0.090 & 0.136 & 0.103 & 0.133 & -0.056 & 0.040 & -0.059 & 0.105 \\
\hline \multirow{4}{*}{$\begin{array}{l}\text { Carotenoid } \\
\left(\mathrm{mg} 100 \mathrm{~g}^{-1}\right)\end{array}$} & I & -0.074 & 0.209 & 0.034 & 0.239 & 0.219 & -0.522 & 0.370 & 0.364 & 0.482 & -0.148 & 0.332 & 0.149 & $0.546^{* *}$ \\
\hline & II & -0.016 & -0.012 & -0.031 & 0.014 & -0.017 & -0.010 & 0.219 & 0.324 & 0.070 & 0.028 & -0.022 & -0.028 & $0.410^{*}$ \\
\hline & III & -0.006 & 0.004 & -0.010 & -0.008 & -0.009 & -0.014 & -0.008 & -0.022 & 0.020 & -0.007 & 0.015 & -0.090 & $-0.481^{* *}$ \\
\hline & P & -0.012 & -0.009 & 0.020 & -0.005 & -0.012 & 0.033 & -0.022 & -0.029 & -0.020 & 0.006 & 0.035 & 0.014 & 0.190 \\
\hline \multirow{4}{*}{$\begin{array}{l}\text { Fibre } \\
(\%)\end{array}$} & I & -0.034 & 0.053 & 0.024 & -0.016 & 0.064 & -0.043 & 0.031 & 0.063 & 0.019 & -0.062 & 0.016 & -0.019 & 0.156 \\
\hline & II & 0.080 & -0.024 & -0.060 & 0.068 & 0.115 & -0.019 & 0.221 & -0.050 & 0.050 & 0.407 & 0.260 & -0.118 & 0.379 \\
\hline & III & 0.017 & -0.005 & -0.021 & 0.004 & 0.041 & -0.015 & 0.023 & 0.017 & 0.009 & -0.058 & -0.012 & 0.021 & -0.194 \\
\hline & P & -0.013 & -0.150 & 0.290 & 0.034 & 0.210 & -0.780 & 0.424 & 0.480 & 0.080 & -1.190 & 0.610 & 0.120 & -0.127 \\
\hline \multirow{4}{*}{$\begin{array}{l}\text { Protein } \\
(\%)\end{array}$} & I & -0.019 & -0.514 & 0.231 & -0.374 & -0.564 & 0.341 & -0.870 & -0.874 & -0.680 & 0.154 & -1.024 & -0.460 & $0.650^{* *}$ \\
\hline & II & -0.103 & -0.060 & -0.130 & 0.079 & 0.024 & 0.143 & 0.263 & 0.289 & 0.079 & -0.170 & -0.270 & -0.026 & 0.084 \\
\hline & III & 0.128 & 0.036 & -0.142 & 0.081 & 0.155 & -0.018 & 0.184 & 0.170 & -0.162 & -0.040 & -0.280 & -0.093 & $-0.619^{* *}$ \\
\hline & P & 0.115 & 0.096 & 0.063 & -0.034 & 0.020 & -0.064 & 0.066 & 0.015 & -0.011 & -0.130 & 0.240 & 0.131 & 0.290 \\
\hline \multirow{4}{*}{$\begin{array}{l}\text { Ascorbic } \\
\text { acid } \\
(\%)\end{array}$} & I & -0.074 & -0.026 & 0.112 & 0.022 & 0.039 & 0.054 & -0.039 & -0.041 & -0.110 & -0.060 & -0.101 & -0.241 & $0.417^{*}$ \\
\hline & II & 0.034 & 0.124 & 0.104 & -0.033 & -0.075 & 0.027 & -0.151 & -0.112 & -0.091 & -0.053 & 0.024 & 0.190 & 0.163 \\
\hline & III & 0.009 & 0.058 & 0.170 & -0.036 & -0.086 & 0.090 & -0.042 & -0.184 & -0.051 & -0.027 & 0.102 & 0.270 & -0.085 \\
\hline & P & -0.155 & -0.341 & -0.170 & 0.104 & 0.234 & -0.089 & 0.391 & 0.326 & 0.129 & 0.085 & -0.353 & -0.749 & 0.188 \\
\hline
\end{tabular}

diameter, chlorophyll $a$, chlorophyll $b$ and protein content (Table 5). Ascorbic acid positively affected yield in $\mathrm{I}^{\text {st }}$ cutting as well as on pooled basis.

\section{Discussion}

Only 1 out of the 3 diploid lines viz. C. album 'Chandanbathua' gave high foliage yield, while the sole exotic tetraploid line (C. album 'Mexico') gave marginally higher yield than the mean value. All the 4 indigenous hexaploid lines performed better than most of the exotic lines giving high yields in both the environments and on overall mean basis.

The higher values of genotypic correlation with respect to their corresponding phenotypic correlations were probably due to the modifier effect of environment on character association at the genetic level. The different cuttings showed low values of residual effect indicating that the characters under study are sufficient to account for variability in the crop. The consistently high positive association between foliage yield and leaf size in all the cuttings and pooled data indicates the utility of this trait for selection with respect to foliage yield. Matteucci (1998) and Sarker et al. (2015) have also reported significant correlation between plant biomass and leaf area in Amaranthus. Such strong association has also been reported in in other crops like coriander, forage maize, cotton and oil palm (Awal et al., 2004; Akram-Ghaderi and Soltani, 2007; Carpici and Celik 2010; Chaulagain et al., 2011). It is evident from Table 4 that leaf size was significantly correlated with chlorophyll $a$ and chlorophyll $b$. This presents a very interesting interwoven relationship. An increase in leaf size would lead to increase in chlorophyll content resulting in higher photosynthesis and in turn enhanced foliage yield. Simultaneously, significant positive correlation of stem diameter with foliage yield plays an important role in enhancing foliage yield, as with large stem diameter, the plant would be more vigorous and bear larger leaves. High genetic correlation between total yield and basal diameter of stalks has also been reported in other vegetable crops
(Lopez-Anido et al., 1997). Leaves/plant was negatively associated with foliage yield and leaf size that suggests that increase in the number of leaves might lead to small leaf size and decreased foliage yield. Earlier, Shukla et al. (2004) have reported significant negative correlation between leaves/plant and foliage yield in Amaranthus tricolor. Significant positive association between leaves/plant and branches/plant has been reported for individual cuttings and for pooled data in vegetable amaranth (Amaranthus tricolor) (Shukla et al., 2004) and is quite understandable, as more branches would lead to more number of leaves/plant. Plant height observed significant positive correlation with branches/plant and leaf size in the II $^{\text {nd }}$ and III $^{\text {rd }}$ cuttings as well as on pooled basis. These results are in conformation with those obtained by Batta et al. (1995) who reported high positive correlation of plant height with leaf area in Amaranthus spp. Despite this, plant height maintained high significant positive association with stem diameter on pooled basis and in II $^{\text {nd }}$ cutting. This suggests that increase in plant height would lead to increase in branches/plant and leaf size along with enhancement in stem girth. This is in accordance with Shukla et al. (2004) who reported close association of plant height with branches/plant and stem diameter. Moisture content had negative correlation with chlorophyll $a$, chlorophyll $b$ and carotenoid content in all the cuttings, except in IId $^{\text {nd }}$ cutting suggesting that increase in moisture leads to decrease in these quality traits. There are reports of the existence of a negative genetic correlation between yield and quality components in forage grasses (Wilkins and Humphreys, 2003; Annicchiarico and Romani, 2005) as well as in cereals (Jenner et al., 1991; Pleijel et al., 1999). It was interesting to note that carotenoid, protein and ascorbic acid had significant positive association with foliage yield only in the $\mathrm{I}^{\mathrm{st}}$ cutting when yield was minimum. The non-significant association of fibre content with foliage yield has also been reported in forage maize (Iptas and Acar, 2006). However, as yield increased with progression of successive cuttings, this association became negative. This is a general expectation since yield is known to be inversely proportional to quality. 
However, in our study, analysis of pooled data revealed that no correlation existed between foliage yield and any of the major quality traits viz. fibre, carotenoid, protein or ascorbic acid. Thus, it is possible to increase yield in C. album without adversely affecting quality of the foliage. Such nonassociation of quality traits with foliage yield has also been reported in vegetable amaranth (Shukla et al., 2004).

Although correlation estimates are helpful in determining the components of complex traits such as yield, they do not provide an exact picture of the relative importance of the component traits (Santos et al., 2014). Correlation coefficients are not sufficient to describe relationship when the causal relationship among traits is needed and may be often misleading due to mutual cancellation of the component traits. Thus, study of path coefficient analysis becomes necessary, which takes into account the causal relationship of the components in addition to the degree of relationship. Path coefficient analysis developed by Wright $(1921,1923)$ permits the separation of correlation coefficient into components of direct and indirect effects and helps the breeder to decide on the use of correlated responses or of selection indices in breeding programs (Dewey and Lu, 1959; Santos et al. 2018). The advantage of path analysis is that it permits the partitioning of correlation coefficient into two components, the first being the path coefficient that measures the direct effect of a predictor variable upon its response variable, and the second is the indirect effect of a predictor variable on the response variable through other predictor variables (Dewey and $\mathrm{Lu}, 1959)$. Therefore, in the present study, genotypic correlations were partitioned into direct and indirect effects to know the relative importance of the components.

Path coefficient analysis was conducted taking foliage yield as dependent variable. Path analysis showed that branches/plant had highest positive direct influence on foliage yield followed by leaf size, moisture content, protein, chlorophyll $a$ and chlorophyll $b$. Both branches/plant and moisture content did not exhibit association with foliage yield due to high negative indirect effect via plant height, ascorbic acid, stem diameter and leaves/plant on branches/plant, and fibre and plant height on moisture content. Branches/plant was also the only trait that exerted positive indirect influence over all other traits. The path analysis also revealed that plant height and stem diameter shared highly negative direct relationship with foliage yield. The correlation analysis, however, revealed significant positive correlation of foliage yield with both plant height and stem diameter due to the presence of positive indirect effect via leaf size and branches/plant. Apart from exerting high negative direct effect, plant height and stem diameter also indirectly influenced most of the traits negatively. Surprisingly, neither chlorophyll $a$ nor chlorophyll $b$ showed high direct or indirect effect on foliage yield. Fibre and ascorbic acid displayed high negative direct effect on yield in the $\mathrm{I}^{\text {st }}$ cutting and on pooled basis. In fact none of the quality traits seemed to majorly influence foliage yield and therefore selection based on quality traits is less likely to lead to yield enhancement in vegetable chenopods. Leaves/plant had high negative direct effect as well as significant negative correlation with foliage yield that makes it logical to select plants having less number of leaves for the improvement of foliage yield. These results are in accordance with those obtained by Shukla et al. (2004) who reported negative correlation of leaves/plant with foliage yield as well as negative direct path value for leaves/plant in vegetable amaranth (A. tricolor L.). Leaf size had high positive direct effect and significant positive association with foliage yield that indicates a true relationship between these traits. Leaf size also indirectly affected foliage yield in a positive direction through majority of other traits. Thus, direct selection for leaf size should be exercised to bring about improvement in foliage yield in C. album.

\section{Conclusions}

This investigation is significant since it is the first such study on correlation and path analysis among foliage yield and different contributing traits in C. album over successive cuttings. The present study has proved that the leaves of $C$. album can serve as an important source of cheap nutrients and successive harvests can be made in this crop that adds to its utility. An important outcome is the conclusion that plant type for increased yield should have less number of large sized leaves.

\section{Acknowledgements}

The authors are thankful to the Director, National Botanical Research Institute, Lucknow, India for providing the facilities and constant encouragement to carry out the present investigation. Atul Bhargava acknowledges C.S.I.R., New Delhi for providing financial assistance.

\section{References}

Abel S, Gislum R, Boelt B (2017). Path and correlation analysis of perennial ryegrass (Lolium perenne L.) seed yield components. Journal of Agronomy and CropScience 203:338-344.

Aletor O, Oshodi AA, Ipinmoroti K (2002). Chemical composition of common leafy vegetables and functional properties of their leaf protein concentrates. Food Chemistry 78:63-68.

Aastveit AH, Aastveit K (1993). Effects of genotype environment interactions on genetic correlations. Theoretical and Applied Genetics 86:1007-1013.

Akram-Ghaderi F, Soltani A (2007). Leaf area relationships to plant vegetative characteristics in cotton (Gosspium hirsutum $\mathrm{L}$.) grown in a temperate sub-humid environment. International Journal of Plant Production 1:63-71.

Annichiarico P, Romani M (2005). Genetic variation, heritability and genetic correlations for forage quality and yield traits of Mediterranean tall fescue germplasm. Plant Breeding 124:99-101.

Awal MA, Ishak W, Endar J, Haniff M (2004). Determination of specific leaf area and leaf area-leaf mass relationship in oil palm plantation. Asian Journal of PlantScience 3:264268.

Bakshi DNG, Sensarma P, Pal DC (1999). A lexicon of medicinal plants of India, Calcutta, Naya Prakash.

Bazile D, Pulvento C, Verniau A, Al-Nusairi MS, Ba D, Breidy J (2016). Worldwide evaluations of quinoa: preliminary results from post 
32

international year of quinoa $\mathrm{FAO}$ projects in nine countries. Frontier in PlantScience 7:850.

Batta RK, Sidhu BS, Mehndiratta PD (1995). Forage production of Amaranthus ssp. on the Indian sub-continent. Rangelands in a sustainable biosphere. Proceedings of the $\mathrm{V}^{\text {th }}$ International Rangeland Congress, Salt Lake City, Utah, USA.

Bhargava A, Shukla S, Katiyar RS, Ohri D (2003a). Selection parameters for genetic improvement in Chenopodium grain on sodic soil. Journal of Applied Horticulture 5:45-48.

Bhargava A, Shukla S, Ohri D (2003b). Genetic association in Chenopodium. Indian Journal of Genetics and Plant Breeding 63:283284.

Bhargava A, Shukla S, Ohri D (2006a). Chenopodium quinoa - an Indian perspective. Industrial Crops Production 23:73-87.

Bhargava A, Shukla S, Ohri D (2006b). Karyotypic studies on some cultivated and wild species of Chenopodium (Chenopodiaceae). Genetic Resources and Crop Evolution 53:1309-1320.

Bhargava A, Shukla S, Ohri D (2007a). Evaluation of foliage yield and leaf quality traits in Chenopodium spp. in multiyear trials. Euphytica 153:199-213.

Bhargava A, Shukla S, Ohri D (2007b). Genetic variability and interrelationship among various morphological and quality traits in quinoa (Chenopodium quinoa Willd.). Field Crops Research 101:104 116.

Bhargava A, Shukla S, Ohri D (2008). Implications of direct and indirect selection parameters for improvement of grain yield and quality components in Chenopodium quinoa Willd. International Journal of Plant Production 2:184-191.

Bhargava A, Shukla S, Ohri D (2010). Mineral composition in foliage of some cultivated and wild species of Chenopodium. Spanish Journal of Agricultural Research 8:371-376.

Bhargava A, Ohri D (2015). Quinoa in the Indian subcontinent. In: Bazile et al. (Eds). FAO and CIRAD: state of the art report of quinoa in the world in 2013. FAO, Rome, Italy pp 511-523.

Bhargava A, Ohri D (2016). Origin of genetic variability and improvement of quinoa (Chenopodium quinoa Willd.). In: Rajpal VR, Rao SR, Raina SN (Eds). Gene pool diversity and crop improvement. Springer International Publishing, Switzerland pp 241-270.

Bilalis D, Roussis I, Fuentes F, Kakabouki I, Travlos I (2017). Organic agriculture and innovative crops under Mediterranean conditions. Notulae Botanicae Horti Agrobotanici Cluj-Napoca 45:323-331.

Bizeti HS, Carvalho CGP, Souza JRP, Destro D (2004). Path analysis under multicollinearity in soybean. Brazilian Archives of Biology and Technology 47:669-676.

CarpiciE, Celik N (2010). Determining possible relationships between yield and yield-related components in forage maize (Zea mays L.) using correlation and path analyses. Notulae Botanicae Horti Agrobotanici Cluj-Napoca 38:280-285.

Chaulagain R, Pant SS, Thapa RB, Sharma MD (2011). Performance of coriander cultivars for green leaf production under late sowing condition. The Journal of Agriculture and Environment 12:67-73.

Chen Y, Lübberstedt T (2010). Molecular basis of trait correlations. Trends in Plant Science 15:454-461.

Dewey DR, Lu KH (1959). A correlation and path coefficient analysis of components of crested wheat grass seed production. Agronomy Journal 51:515-518.

dos Santos A, Ceccon G, Davide LMC, Correa AM, Alves VB (2014). Correlations and path analysis of yield components in cowpea. Crop Breeding and Applied Biotechnology 14:82-87.

Falconer DS (1989). Introduction to quantitative genetics. ( $\left.3^{\text {rd }} \mathrm{ed}\right)$ London, Longman Sci. and Tech.

Fuentes F, Bhargava A (2011). Morphological analysis of quinoa germplasm grown under lowland desert conditions. Journal of Agronomy and CropScience 197:124-134.

Fuentes F, Paredes-Gónzalez X(2015). Nutraceutical perspectives of quinoa: biological properties and functional applications. In: Bazile et al. (Eds). FAO and CIRAD: state of the art report of quinoa in the world in 2013. FAO, Rome, Italy pp 286-299.

Garcia del Morel LF, Rharrabti Y, Villegas D, Royo C (2003). Evaluation of grain yield and its components in durum wheat under Mediterranean conditions: an ontogenic approach. Agronomy Journal 95:266-274.

Glick D (1954). Methods of biochemical analysis. Vol. 1, New York, Interscience Publishers Inc.

Iptas S, Acar AA (2006). Effect of hybrid and row spacing on maize forage yield and quality. PlantSoil andEnvironment 11:515-522.

Jacobsen SE (2003). The worldwide potential for quinoa (Chenopodium quinoa Willd.). Food Reviews International 19:167-177.

Jenner CF, Ugalde TD, Aspinall D (1991). The physiology of starch and protein deposition in the endosperm of wheat. Australian Journal of Plant Physiology 18:211-226.

Jensen A (1978). Chlorophylls and carotenoids. In: Hellebust JA, Craigie JS (Eds). Handbook of physiological methods: physiological and biochemical methods, Cambridge University Press, Cambridge, pp 570.

Johnson HW, Robinson HF, Comstock RE (1955). Genotypic and phenotypic correlation in soybean and their implication in selection. AgronomyJournal 47:477-485.

Joshi BD (1991). Genetic resources of leaf and grain Amaranthus and chenopod. In: Swaminathan MS, Jana S (Eds). Biodiversity. Macmillan IndiaLtd, Madras pp 121-134.

Kaul HP, Laible WB, Nalborczyk E, Pirog S, Wasiak K (1996). The suitability of amaranth genotypes for grain and fodder use in central Europe. Bodenkultur 47:173-181.

Khan ASMMR, Eyasmin R, Rashid MH, Ishtiaque S, Chaki AK (2016). Variability, heritability, character association, path analysis and morphological diversity in snake gourd. Agriculture and Natural Resources 50:483-489.

Kirtikar KR, Basu BD (2001). Indian medicinal plants (Vol. 9), Dehradun, Oriental Enterprises.

Kunkel G (1984). Plants for human consumption. Koeltz Scientific Books, Koenigstein.

Lopez-Anido F, Cointry E, Picardi L, Camadro E (1997) Genetic variability of productive and vegetative characters in Asparagus officinalis L.estimates of heritability and genetic correlations. Brazilian Journal of Genetics 20:275-281. 
Lowry OH, Rosebrough NJ, Farr AL, Randall RJ (1951). Protein measurement with the folin-phenol reagent. Journal of Biological Chemistry 193:265-275.

Madrid D, Salgado E, Verdugo G, Olguín P, Bilalis D, Fuentes F (2018). Morphological traits defining breeding criteria for coastal quinoa in Chile. Notulae Botanicae Horti Agrobotanici Cluj-Napoca 46:190196.

Manenti T, Sørensen JG, Moghadam NN, Loeschcke V (2016). Few genetic and environmental correlations between life history and stress resistance traits affect adaptation to fluctuating thermal regimes. Heredity 117:149-154.

Matteucci SD (1998). Potencial productivo del amaranto en la pampa onduLada, Argentina: comportamiento de seis germoplasmas. Revista dela Facultad de Agronomía 15:560-570.

Mihretu Y, Weyessa G, Adugna D (2014). Variability and association of quantitative characters among okra (Abelmoschus esculentus (L.) Moench) collection in South Western Ethiopia. Journal of Biological Sciences 14:336-342.

Moerman D (1998). Native American ethnobotany. Timber Press, Oregon.

Partap T (1990). Exploiting underexploited crop plants of mountain agriculture: chenopods. In: Riley KW, Mateo N, Hawtin GC, Yadav $\mathrm{RP}(\mathrm{Eds})$. Mountain agriculture and crop genetic resources, Oxford and IBH,New Delhi.

Partap T, Kapoor P (1985). The Himalayan grain chenopods I. distribution and ethnobotany. Agriculture Ecosystem and Environment 14:185199.

Partap T, Joshi BD, Galwey NW (1998). Chenopods: Chenopodium spp. promoting the conservation and use of underutilized and neglected crops. Institute of Plant Genetics and Crop Plant Research, Gatersleben/ International Plant Genetic Resources Institute, Rome, Italy.

Pleijel H, Mortensen L, Fuhrer J, Ojanpera K, Danielsson H (1999). Grain protein accumulation in relation to grain yield of spring wheat (Triticum aestivum L.) grown in open-top chambers with different concentrations of ozone, carbon dioxide and water availability. Agriculture Ecosystems and Environment 72:265-270.

Prakash D, Nath P, Pal M (1993). Composition, variation of nutritional contents in leaves, seed protein, fat and fatty acid profile of Chenopodium species. International Journal of Food Science and Agriculture 62:203205.

Punzalan D, Delcourt M, Rundle HD (2014). Comparing the intersex genetic correlation for fitness across novel environments in the fruit fly, Drosophila serrata. Heredity 112:143-148.

Ranjbar A, Sepaskhah AR, Emadi S (2015). Relationships between wheat yield, yield components and physico-chemical properties of soil under rain-fed conditions. International Journal of Plant Production 9:433466.

Risi J, Galwey NW (1989). The pattern of genetic diversity in the Andean grain crop quinoa (Chenopodium quinoa Willd.). II Multivariate methods. Euphytica41:135-145.
Santos A, Ceccon G, Davide LMC, Correa AM, Alves VB (2014). Correlations and path analysis of yield components in cowpea. Crop Breeding and Applied Biotechnology 14:82-87.

Santos EAd, Almeida A-AFd, Branco MCdS, Santos ICd, Ahnert D, Baligar VC, Valle RR (2018). Path analysis of phenotypic traits in young cacao plants under drought conditions. PLoS One 13:e0191847.

Sarker U, Islam MT, Rabbani MG, Oba S (2015). Variability, heritability and genetic association in vegetable amaranth (Amaranthus tricolor L.). Spanish Journal of Agricultural Research 13:1-8.

Shukla S, Bhargava A, Chatterjee A, Singh SP (2004). Interrelationship among foliage yield and its contributing traits in vegetable amaranth $(A$. tricolor). Progressive Horticulture 36:299-305.

Shukla S, Bhargava A, Chatterjee A, Singh SP (2006) Genotypic variability in vegetable amaranth (Amaranthus tricolor $\mathrm{L}$.) for foliage yield and its contributing traits over successive cuttings and years. Euphytica 151:103110.

Sincik M, Goksoy A (2014). Investigation of correlation between traits and path analysis of confectionary sunflower genotypes. Notulae Botanicae Horti Agrobotanici Cluj-Napoca 42:227-231.

Siddiqi A, Shukla S, Rastogi A, Bhargava A, Niranjan A, Lehri A (2016). Relationship among phenotypic and quality traits in indigenous and exotic accessions of linseed. Pesquisa Agropecuária Brasileira 51:1964 1972.

Singh RK, Chaudhary BD (1985). Biometrical methods in quantitative genetic analysis, New Delhi, Kalyani Publishers.

Singh VK, Govil JN, Hashmi S, Singh G (2003). Recent progress in medicinal plants (Vol. 7) ethnomedicine and pharmacognosy. LLC, Studium Press.

Solovieff N, Cotsapas C, Lee PH, Purcell SM, Smoller JW (2013). Pleiotropy in complex traits: challenges and strategies. Nature Reviews Genetics 14:483-495.

Streiner DL (2005). Finding our way: an introduction to path analysis. Canadian Journal of Psychiatry 50:115-122.

Tanaka T (1976). Tanaka's cyclopaedia of edible plants of the world. Keigaku Publishing, Tokyo.

Watson CA (1994). Official and standardized methods of analysis. The Royal Society of Chemistry, Cambridge.

Wilkins PW, Humphreys MO (2003). Progress in breeding perennial forage grasses for temperate agriculture. Journal of Agricultural Science 140:129-150.

Wright S (1921). Correlation and causation. Journal of Agricultural Research 20:557-585.

Wright $S$ (1923). The theory of path coefficients: a reply to Niles's criticism. Genetics 8:39-255.

Young KJ, Eunji L, Seungkook P, Won CG, Kwon BN (2000). Physicochemical quality characteristics of several Chinese cabbage (Brassica pekinensis) cultivars. Korean Journal of Horticultural Science and Technology 18:348-352. 